\title{
INSAR Flat-earth Phase Removal Approach Based on DEM to Settlement Area
}

\author{
Xing-Tong CHEN, Xiao-Feng ZHU, Xiao-Hong WANG, and Yang Cai \\ North China University of Science and Technology,Hebei,Tangshan,063009, China \\ 1604464340@qq.com
}

\begin{abstract}
The difficulties of phase unwrapping was increased with the existence of flat-earth phase removal, at the same time, it covered up the real topographical conditions. In order to solve this problem , the paper presented a method of re-flattening. based on DEM to eliminate flat-earth phase, with the interferogram, and then chose some special control points to construct polynomial fitting, removed residual phase and noise. By analyzing the ALOS/PALSAR at the mine subsidence area, the contrast experiment was carried out. The results showed that the method can clearly reflect the earth's surface changes, reduce the surface deformation error, get a more accurate interferogram phase and improve the precision of the INSAR interferometry.
\end{abstract}

Synthetic Aperture Radar Interferometry (INSAR)measurement technique combined the synthetic aperture radar imaging theory and electromagnetic interference technology, using the observations of two or more SAR image complex data in the same area to process coherence to obtain more precise digital elevation model and more accurate millimeter surface deformation information[1]. However, Flat-earth phase removal had a very important effect on the entire INSAR data. Due to the using of synthetic aperture radar imaging with slant range way. Flat-earth phase removal appeared, so the distance was different when the radar echo signal propagated, and phase position changed which should remain unchanged at the same height in the interference figure, the phenomenon also showed that the interferogram presented periodically interference fringes.The presence of flat-earth phase removal obscured the change of interference fringes, so the interferogram can not reflect the real situation of geomorphology, and made fringes intensive, so the difficulty of phase unwrapping increased. There fore, before unwrapping the interferogram, the flat-earth phase must be removed. Approaching to common flat-earth phase removal were mainly the following methods[2-3]: (1) A method of flat-earth phase removal based on orbit parameters was to eliminate flat-earth phase that the reference ellipsoid caused by the position and orbital parameters of the imaging area. The principle of the method was simple and high precision theory but lowering efficiency, which depended on the accuracy of the orbit position.(2) Frequency shift method had a small amount of calculation and no other additional conditions merit. Tocalculate the flat phase by frequency interference fringes, eliminate the flat-earth phase. It was more effective for flat areas, But for the settlement of the surface area, the fringe frequency that some part of terrain changes was as same as peaceful fringes of the frequency, if it was removed. It was likely to cause an error, and can notaccurately eliminate the influence of the flat phase. (3) Flat-earth phase removal based on DEM method can achieve high elevation accuracy under the conditions of little parameter data. However, this method was influenced by abnormal phase and phase noise, made some difficult terrain of the interferogram which was still not clearly reflected. Based on DEM data to eliminate the flat-earth phase, This paper presented a method of re-flattening to improve accuracy.

\section{Geometric principles of flat-earth phase removal}

According to INSAR imaging techniques, as shown in figure 1, the figure had two points of the level terrain: $\mathrm{P} 1$ and $\mathrm{P} 2$. the heights of points $\mathrm{P} 1$ and $\mathrm{P} 2$ are same. In the interference pattern the phase difference between $\mathrm{P} 1$ and $\mathrm{P} 2$ was $\Delta \Phi[4]$, as in

$$
\Delta \Phi=\frac{4 \pi B_{\perp} \Delta r_{s}}{\lambda r_{0} \tan \theta}
$$

Where ${ }^{B_{\perp}}$ was expressed as the vertical baseline; B was expressed as the distance between the antennas; $\Delta \mathrm{r}_{\mathrm{s}}$ was expressed as the slope distance difference of $\mathrm{P} 1$ and P2 points; $\theta$ was expressed as the look-angle of radar; r0was expressed as the initial slope distance length.

As it can be seen from the equation (1) the ground surface without elevation variation also generated linear varied interference phase. so the specific geometry of 
INSAR interferometer system leaded to the existence of the flat-earth phase. For the the different range positions and the exact same point, height of which was different from the interferogram phase. Not only the flat-earth phase existed in the range, but the satellite orbit was not perfectly parallel, the earth was irregular ellipsoid, there also existed in the azimuth direction. According to the equation (1), the common method of flat-earth phase removal calculated the relative change in the flat-earth phase of the scene to realize the purpose.

To eliminate the flat-earth phase of the range, multiplied the complex intensity of each pixel in the interference pattern with the complex exponential related slant distance phase. it was possible to correct the impact of flat-earth phase[5]:

$$
\beta=\exp \left(-i \frac{4 \pi B_{\perp} \Delta r_{s}}{\lambda r_{0} \tan \theta}\right)
$$

The interference phase difference became :

$$
\Delta \Phi=\frac{4 \pi B_{\perp} \Delta z}{\lambda r_{0} \sin \theta}
$$

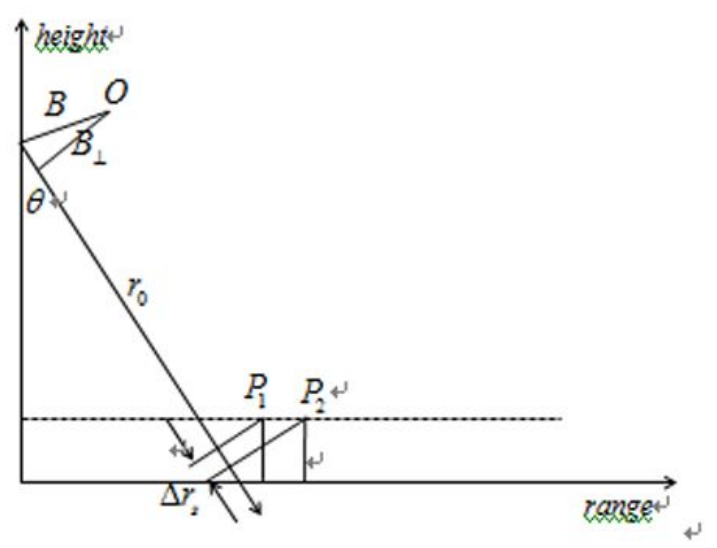

Figure 1.The Diagram of Interferogram Phase Changes With The Slant range geometry

Where $\Delta z$ was the actual height difference of the terrain. As can be seen from the above equation, after phase compensation, the phase can directly reflect the topology of the terrain height.

\section{Flat-earth Phase Removal Based on DEM Method}

Flat-earth phase removal based on DEM, the first step was to get DEM data in the study area. This paper used a global DEM data to eliminate flat-earth phase, it can easily get DEM data in the study area, such as a resolution of about $90 \mathrm{~m}$ of the SRTM DEM, the reference coordinate system was WGS84 coordinate system. Specific methods using DEM data to eliminate flat-earth phase was as follows:

\subsection{DEM Resampling}

Read and calculate the interval of the midpoint between the DEM, while calculating intervals of the element points in the interference picture and their ratio. According to the ratio between the interference pattern and the DEM, proceeded resample for the DEM point, and bilinear interpolation method was used to obtain its pixel elevation value. The expression for the bilinear interpolation [6]:

$$
\begin{aligned}
& f(i+u, j+v)=(1-u)(1-v) f(i, j)+(1-u) v f(i, j+1) \\
& +u(1-v) f(i+1, j)+u v f(i+1, j+1)
\end{aligned}
$$

Where $\mathrm{f}(*, *)$ denoted a pixel gray values, $\mathrm{u}$ and $\mathrm{v}$ were non-integers.

\subsection{Flat-earth Phase Removal}

According to the oblique projection equations of the Doppler equation, nonlinear equations and ellipsoid equation posed, by using of orbital parameters, the DEM data was converted to geocentric coordinate system. By orbit interpolation and bilinear interpolation, the DEM data was converted into phase values, and then subtracted reference value calculated DEM data from the interferogram fringe phase, it achieved the purpose of flat-earth phase removal.

\subsection{Re-flattening}

Since the interference patterns till existed abnormal phase and phase noise caused by orbit parameters, the phenomena madeflat-earth phase removal not completely, to further Refinement and Re-flattening. After the interferogram, selecting some correlation coefficient greater than 0.7 control points from figure 2, which facilitated extraction of elevation, and control point were selected relatively flat and stable place. The distribution of control points was not too scattered. The number of control points were less than 7, the error range was less than one pixel resolution. The selected control points data can see in table 1 .

By using the selected point setting orbit correction parameters, recalculated the satellite position, and then calculated the points on the datum to the distance of the satellite, and then get the corresponding points of the flat phase. In the interferogram, by subtracting the datum from the interferogram fringe phase, it achieved the purpose of Re-flattening 
Table 1. Control Point Statistics

\begin{tabular}{|l|l|}
\hline Number & Correlation Coefficient \\
\hline 1 & 0.798 \\
\hline 2 & 0.781 \\
\hline 3 & 0.791 \\
\hline 4 & 0.848 \\
\hline 5 & 0.767 \\
\hline 6 & 0.751 \\
\hline 7 & 0.856 \\
\hline 8 & 0.843 \\
\hline 9 & 0.726 \\
\hline
\end{tabular}

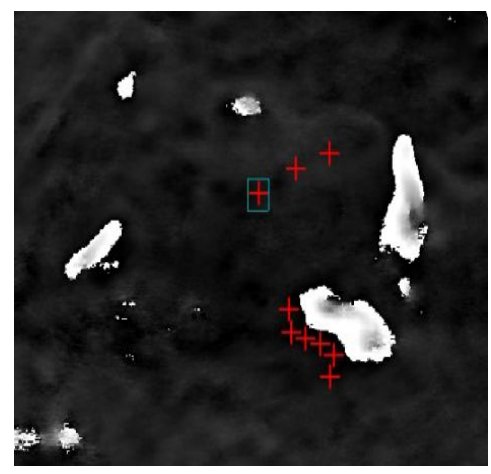

Figure 2. Control Points Distribution

The number of polynomial coefficients was used to remove residual phase, the degrees of polynomial was used to estimate the phase slope, If the number of coefficients was higher, the control points inputting will automatically decreased, The default value was 3 , which indicated phase ramp in the range and azimuth direction which added a constant phase to correct, its polynomial expression was:

$$
\begin{aligned}
& \phi=k_{1}+k_{2} x+k_{3} y+k_{4} x^{2}+k_{5} x y \\
& +k_{6} y^{2}+k_{7} x^{3}+k_{8} x^{2} y+k_{9} x y^{2}+k_{10} y^{3}
\end{aligned}
$$

\section{Experimental analysis}

\subsection{Experimental Area and the Experimental data}

The area of surface subsidence was as the experimental area. ALOS/PALSAR images were chosen to processed interference experiments. External DEM data was obtained by using the EV-INSAR.

Since the surface subsidence area was complex, the baseline data would be estimated before the experiment. the purpose was to determine whether data can be interference from time baseline and space baseline. The long spatial baseline was better in INSAR data processing, and it was best if it was less than $1 / 3$ of the critical baseline. Time baseline of master and slave images was $90 \mathrm{~d}$ in the experimental data area. space baseline was $661.798 \mathrm{~m}$, and critical baseline was between $-6196.108 \mathrm{~m}$ and $6196.108 \mathrm{~m}$, it conformed to interference condition for INSAR data.

\subsection{Experimental Results and analysis}

Two scene Spaceborne INSAR experimental data was get, by using the DEM data to remove flat-earth phase, selecting WGS84 model as a reference to remove flat-earth phase. Comparison of two methods of extracting DEM image was given. After the master and slave images make coregistration in the study area, the plurality of master and slave images corresponding pixels proceeds conjugate multiplier to obtain the interferogram, figure 3 was obtained interference phase. As can be seen from the figure 3, Regular interference fringes covered up the real terrain, fringes were displayed, because correlation coefficient was relatively poor. Interference fringe spacing expressed the altitude difference, by means of the baseline estimate, and its altitude difference was $93.048 \mathrm{~m}$. Interference phase ranges from - $л$ to $л$. the interference fringe showed dense and diagonal. Its characteristics were not parallel to the fringe direction of the range and azimuth, the flat-earth phase existed mainly direction of the range.It was mainly due to the two parallel orbits. Therefore, the range and azimuth have to remove the flat-earth phase.

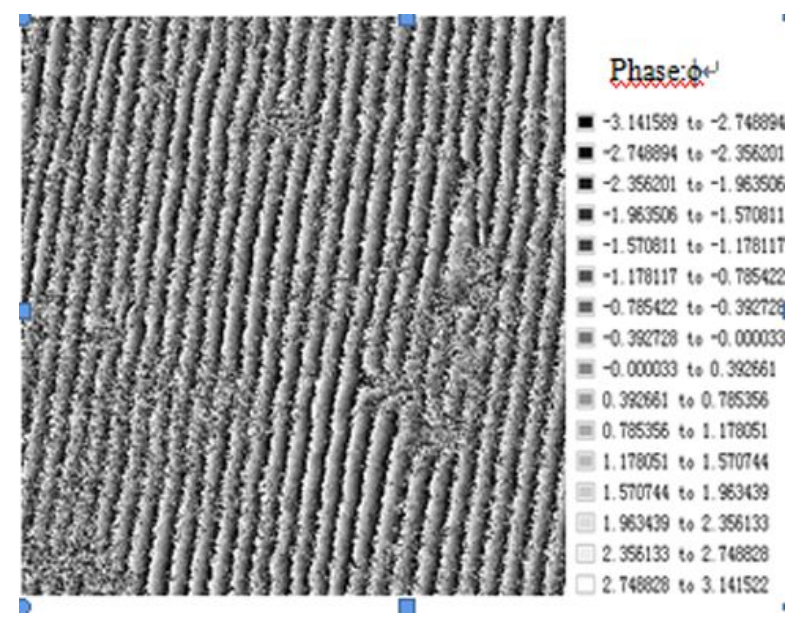

Figure 3.The Interference Phase Image

Figure 4 was the interference phase after removing flat-earth phase. It used the DEM data to remove the flat-earth phase. Interference fringe Substantially removed in the figure, it showed the flat-earth phase had been removed from the direction of range and azimuth. Interferogram can reflect changes in the terrain. However, due to the effects of noise and residual phase, Unwrapped phase do not correctly translate into deformation value. So it was necessary to re-flattening. 


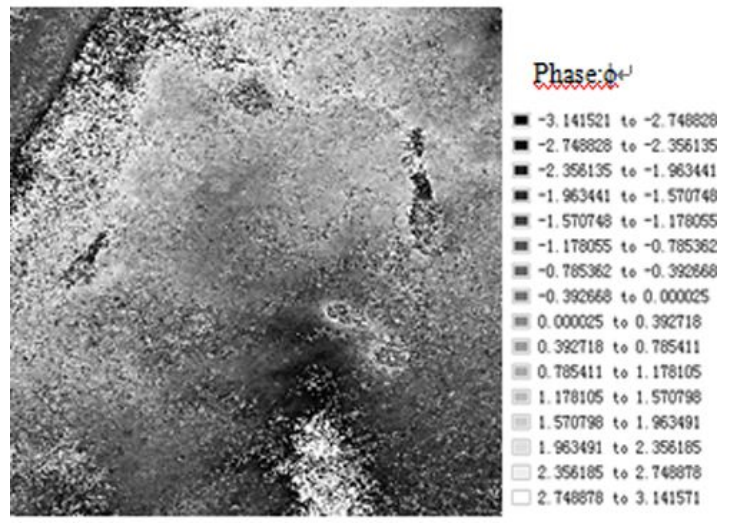

Figure 4. The Interference Image of Flat-earth phase Removal

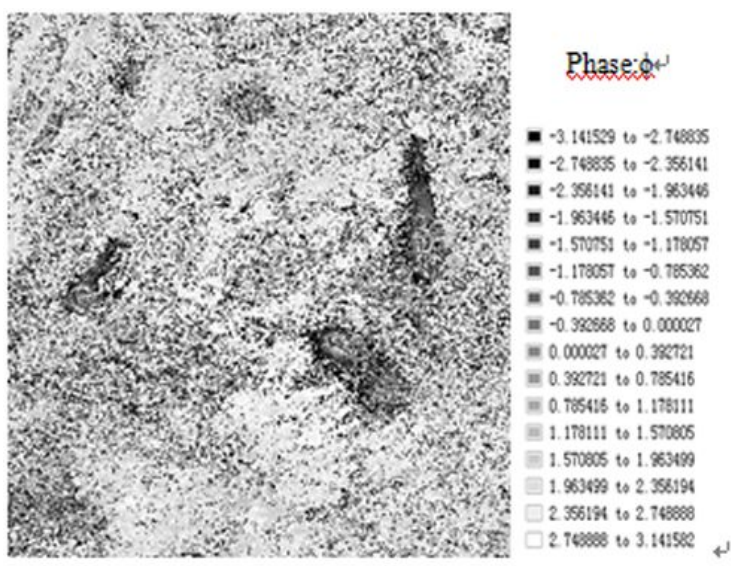

Figure 5.The Interference Phase Image of Re-flattening

Figure 5 was the interference phase after re-flattening. Compared withfigure4, figure 5 interferogram phase information was more obvious, the invisible levels became more clear before re-flattening. Interferogram can clearly see the terrain change information.

In order to demonstrate consistent visual and quantitative evaluation of the results of the evaluation results, this paper quantitatively analyzed from the surface deformation and phase standarddeviation. It more fully and objectively showed that the method was effective.

Figure 6 was the surface deformation image by flat-earth phaseremoval based on DEM method. Figure 7 was the surface deformation image by the method of re-flattening. Comparing figure 6 and figure 7,it can be seen, the surface deformation was generally lower in the same area, maximum deformation reached $0.666146 \mathrm{~m}$. By refinement and noise removal, the highly data error by this method slowly reduced $\Delta \mathrm{z}$ approaches terrain changed.

Comparing with flat-earth phase removal based on DEM method, re-flattening method removed better phase noise. According to the indicator of quantitative evaluation phase standard deviation, the size of the standard deviation reflected dynamic range of phase amplitude. As can be seen from Table 2, Phase standard deviation became1.2878 to 1.4638 ,increasedby0.176. It suggested that standard deviation of the phase changed small, the method maintained the dynamic range of phase amplitude.

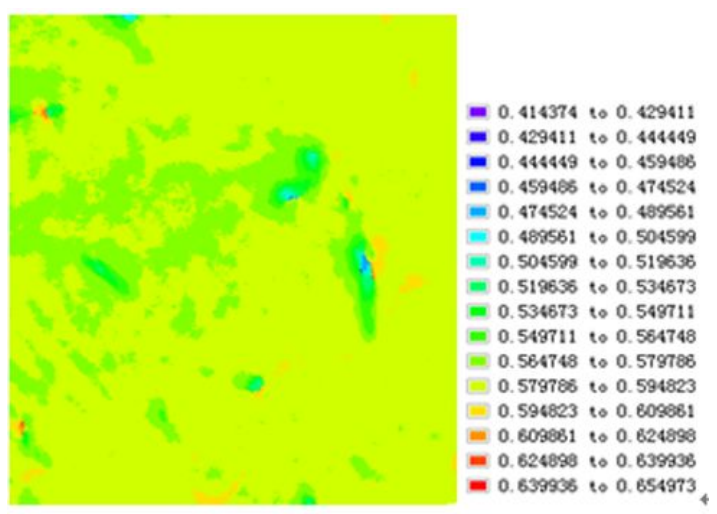

Figure 6.The Surface Deformation of Flat-earth Phase Removal Image(unit: $\mathrm{m}$ )

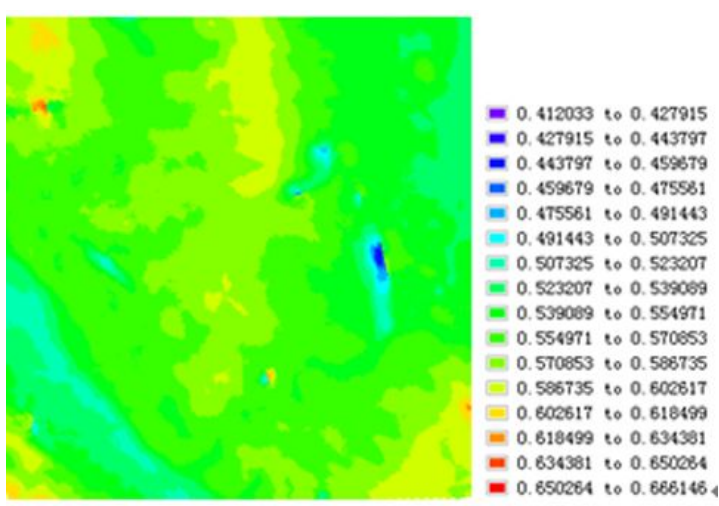

Figure 7.The Surface Deformation of Re-flattening(unit: m)

Table 2.Two Methods Have The Impact of Standard Deviation

\begin{tabular}{|c|l|}
\hline methods & \multicolumn{1}{|c|}{ Standard deviation/rad } \\
\hline $\begin{array}{c}\text { Flat-earth phase removal } \\
\text { based on DEM method }\end{array}$ & 1.2878 \\
\hline Re-flattening method & 1.4638 \\
\hline
\end{tabular}

\section{Summary}

By analyzing the ALOS/PALSAR actual experimental data and the original DEM data, it showed that the purpose of the removal re-flattening method can be achieved with DEM data. Constructing polynomial fitting can make DEM terrain's change more obvious, the accuracy of elevation improved effectively, and reduce the surface elevation error. Experimental results showed this method was very effective. It had important significance on improving the accuracy of DEM reconstruction and reducing the complexity level of the INSAR data processing. By using INSAR technology to extract DEM, it had all-weather, high accuracy of extracting DEM data, fast speed and other characteristics, it can solve very difficult problem that the conventional method while extracting DEM.INSAR technology provided more efficient, cost-effective new method for the subsidence monitoring, and had good application prospects. 


\section{References}

1. Hanssen RF.Radar Interferometry Data Interpretation and Error Analysis[M].New York: Kluwer Academic Publishers(2002).

2. Hong-An W, Yi-Xian T, Hong Z, Chao W. Flat-Phase Removal for ALOS/PALSAR Interferometry Based on Orbit State Vectors[J]. Geomatics and Information Science of Wuhan University, 35(1):91-96(2010).

3. Zhi-Ming L, Xiao-Tao T. Baseline Estimation and Flat Earth Removal ofERS-1/2 Data Based on Orbital Parameter[J]. Geomatic Science and Engineering, 24(1):26-28(2004).
4. Fen-Fen H, Ji-Xian Z, Ke-Zhong D. Comparative Study on Several Methods of Removing the Flat-earth Effect in Airborne InSAR System[J]. Remote Sensing Information, 5:57-61(2010).

5. Lei Y, Yong-Jun Z, Zhi-Gang W. Frequency Shifted-based Approach for InSAR Flat Earth Effect Removal[J]. Journal of Electronic Measurement and Instrument, 18(4):15-20.(2004).

6. Cai D. Research of Image Interpolation Algorithm. $\mathrm{Ph}$ D Thesis. Chongqing: Chongqing University(2011). 